\title{
COVID-19 pneumonia during long-term migraine prophylaxis with fremanezumab: a case report
}

\author{
Luigi Francesco lannone ${ }^{1} \cdot$ Pierangelo Geppetti $^{1} \cdot$ Alberto Chiarugi $^{1} \cdot$ Francesco De Cesaris $^{1}$ (i)
}

Received: 19 May 2021 / Accepted: 3 June 2021 / Published online: 12 June 2021

c) Società Italiana di Medicina Interna (SIMI) 2021

\section{Dear Editor,}

Migraine is a subtype of primary headache with a prevalence of $14-16 \%$ worldwide and is a leading cause of global disability under 50 years of age. Small molecule receptor antagonists (gepants), or monoclonal antibodies (mAbs) against calcitonin gene-related peptide (CGRP), have recently become available for migraine prophylaxis with proven efficacy. Although this new class of biologicals has shown a remarkable tolerability profile, their safety in the general population and under concomitant specific pathological conditions remains to be established. CGRP is highly represented in afferent nerve fibers in the lung, and seems to have a role in vasodilation of pulmonary vessels and in the attenuation of chronic hypoxia $[1,2]$. On this basis, it has been hypothesized that CGRP receptor antagonism could be associated with cardiopulmonary adverse events in patients with COVID-19 [3]. Here, for the first-time, we report the outcome of a patient chronically treated (21 months) with the anti-CGRP antibody fremanezumab for migraine prophylaxis who developed mild COVID-19-related pneumonia.

A 52-year-old female nurse had personal history of ovariectomy due to endometriosis, uterine polypectomy, head trauma without loss of awareness during adolescence, and no other relevant disorders. Family history positive for migraine (i.e., daughter) and no other relevant diseases.

The patient reported headache insurgence at the age of six and fulfilled the current diagnostic criteria for high frequency (8-14 attacks/ month) migraine without aura according to ICHD-3. Attacks consisted of severe, right hemicrania pulsating pain, usually accompanied by phonophobia, photophobia, and need to rest. Attacks were treated with triptans

Francesco De Cesaris

francesco.decesaris@unifi.it

1 Headache Center and Clinical Pharmacology, Azienda Ospedaliero Universitaria Careggi, Largo Brambilla 3, 50139 Florence, Italy (i.e., zolmitriptan $2.5 \mathrm{mg}$ ) often with full pain relief. Prior preventive therapies included propranolol and amitriptyline without advantage. In 2018, she was enrolled in a clinical trial with fremanezumab. The initial randomized 3-month period was followed by a 9-month open label phase, during which she showed excellent response and no adverse events. At the end of the clinical trial, fremanezumab $(225 \mathrm{mg}$ monthly) treatment was maintained for migraine prophylaxis for 9 months, reporting a headache frequency of $<3$ attacks per month and good tolerability.

In March 2020, while under fremanezumab treatment, the patient referred to the emergency room for flu symptoms. Presenting symptoms were non-productive cough, fever $\left(>37.5^{\circ} \mathrm{C}\right.$ ), generalized weakness, and headache, followed by severe asthenia. An interstitial right pneumonia was evidenced with X-ray imaging, compatible with COVID-19. Reverse transcriptase polymerase chain reaction (RT-PCR) swab testing was positive, confirming SARS-Cov-2 infection. General and neurological examinations were normal with blood oxygen saturation $\geq 99 \%$.

Complete blood workup was in range, excluding reactive C-protein $(7.58 \mathrm{mg} / \mathrm{dl}$; reference value, $\mathrm{RV}<0.5)$ and white blood cell count $\left(3700 \mathrm{~mm}^{3}\right.$; RV 4000-11,000). Considering the overall health conditions, the absence of dyspnea and other symptoms related to pneumonia, and the emergency situation due to the epidemic outbreak, physicians proposed house quarantine. Treatment prescription was limited to $1000 \mathrm{mg}$ acetaminophen on need, and periodically checking blood saturation. Fremanezumab was not discontinued. Symptoms rapidly withdrew one week after hospital admission, and two RT-PCR swabs resulted negative after one month.

Monoclonal anti-CGRP antibodies have recently been approved in the European Union for the prophylaxis of high frequency episodic and chronic migraine [4]. Their efficacy, general tolerability, and compliance associated with monthly administration, have favored their use. However, due to the ubiquitous distribution of CGRP, including 
in the pulmonary vasculature, and its protective role in systemic arterial hypertension, heart failure, pulmonary hypertension and inflammation, concerns about potential detrimental effects of long-term blockade of CGRP have been raised in COVID-19 patients with associated pulmonary and cardiovascular complications $[3,5,6]$. On the other hand, small molecule CGRP receptor antagonists have been proposed for COVID-19 treatment. A phase-II trial to test the efficacy of intranasal zavegepant, a thirdgeneration CGRP receptor antagonist [7], in hospitalized patients with COVID-19 infection to reduce lung inflammation has been authorized by the Food and Drug Administration (FDA) (NCT04346615) [8]. The rationale is based on preclinical evidence that CGRP increases pro-inflammatory cytokine (e.g., interleukine-6 [IL-6]) production, and $\mathrm{T}$ cell polarization into Th17 lymphocytes), two responses considered of relevance to COVID19 infection $[9,10]$. However, it is still unknown whether the basal or increased release of CGRP plays a role in regulating the pulmonary and cardiovascular functions in COVID-19 patients. In this regard, it is worth noting that a recent study indicates a reduced concentration of plasmatic CGRP in patients with COVID-19 infection [11].

Thus, the impact of drugs targeting the CGRP signaling pathway in the cardiopulmonary functions of COVID-19 patients remains controversial. No clinical studies have been reported on this topic, and only one case report has been published so far, showing no adverse events related to various treatments, including 1-year treatment with fremanezumab in a patient with suspected COVID-19 related meningoencephalitis [12]. Our report, showing that very long-term treatment (21 months) with fremanezumab $225 \mathrm{mg} / \mathrm{monthly}$ does not worsen mild pneumonia associated with SARS-Cov-2 infection in a patient who achieved a rapid and complete resolution without sequelae, appears of relevance to both CGRP and COVID-19 pathophysiology. It is also worth noting that, despite the concomitant illness, the frequency of headache attacks was unchanged. Due to the high frequency of headache in COVID-19 patients and the persistent headache symptomatology as a possible chronic sequela of the infection [13, 14], the general safety of fremanezumab in the present case could encourage the continuation of treatments with anti-CGRP molecules in these patients.

To our knowledge, this is the second case of a patient being treated with a monoclonal anti-CGRP antibody during the SARS-Cov-2 infection [12]. It is, however, the first case that reports a very long-term therapy in a patient with COVID-19-associated pneumonia. Prolonged CGRP blockade does not necessarily worsen cardiovascular/pulmonary complications COVID-19-related but the possible effect of prolonged CGRP blockade needs to be investigated. Further studies are required to assess the safety of anti-CGRP remedies in COVID-19 patients, and their possible beneficial effects.

Funding The authors received no financial support for the research, authorship and/or publication of this article.

\section{Declarations}

Conflict of interest P.G: received personal fees from Allergan, Eli Lilly, Novartis, Amgen, TEVA; Grants from Amgen, TEVA, Eli Lilly, Allergan, Chiesi; Scientific Advisory Board, Endosome Therapeutics; Founding scientist of FloNext srl, Spinoff of the University of Florence. F.D.C received personal fees from TEVA, Eli Lilly, Novartis. Other authors have no conflicting interests.

Human and animal rights This article does not contain any studies with human or animal subjects performed by the any of the authors.

Informed consent Informed consent was obtained from the patient to utilize her medical records for the purpose of this report. All information has been appropriately anonymized.

\section{References}

1. Lange M, Enkhbaatar P, Traber DL et al (2009) Role of calcitonin gene-related peptide (CGRP) in ovine burn and smoke inhalation injury. J Appl Physiol (1985). 107(1):176-84. https://doi.org/10. 1152/japplphysiol.00094.2009

2. Champion HC, Bivalacqua TJ, Toyoda $K$ et al (2000) In vivo gene transfer of prepro-calcitonin gene-related peptide to the lung attenuates chronic hypoxia-induced pulmonary hypertension in the mouse. Circulation 101(8):923-930. https://doi.org/10.1161/ 01.CIR.101.8.923

3. Skaria T, Walchli T, Vogel J (2021) CGRP receptor antagonism in COVID-19: potential cardiopulmonary adverse effects. Trends Mol Med 27(1):7-10. https://doi.org/10.1016/j.molmed.2020.10. 005

4. Sacco S, Bendtsen L, Ashina M et al (2019) European headache federation guideline on the use of monoclonal antibodies acting on the calcitonin gene related peptide or its receptor for migraine prevention. J Headache Pain 20(1):6. https://doi.org/10.1186/ s10194-018-0955-y

5. Robertson CE (2020) Could CGRP antagonists be helpful in the fight against COVID-19? Headache 60(7):1450-1452. https://doi. org/10.1111/head.13853

6. Joshi N, McAree M, Klimowich K et al (2020) Oral candidiasis in a migraine patient taking erenumab and galcanezumab: a case report. SN Comprehens Clin Med 2:658-661. https://doi.org/10. 1007/s42399-020-00300-5

7. Cipolla F, Capi M, Lionetto L et al (2021) Zavegepant. Calcitonin gene-related peptide (CGRP) receptor antagonist, treatment of migraine. Drugs Fut. 46:281. https://doi.org/10.1358/dof.2021. 46.4.3238324

8. Safety and efficacy trial of zavegepant intranasal for hospitalized patients with COVID-19 requiring supplemental oxygen - full text view - ClinicalTrials.gov. 2021.

9. Cohen JM, Ning X, Kessler Y, Rasamoelisolo M, Campos VR, Seminerio MJ et al (2021) Immunogenicity of biologic therapies 
for migraine: a review of current evidence. J Headache Pain 22(1):3. https://doi.org/10.1186/s10194-020-01211-5

10. Sakuta H, Inaba K, Muramatsu S (1995) Calcitonin gene-related peptide enhances cytokine-induced IL-6 production by fibroblasts. Cell Immunol 165(1):20-25. https://doi.org/10.1006/cimm.1995. 1182

11. Ochoa-Callejero L, Garcia-Sanmartin J, Villoslada-Blanco P et al (2021) Circulating levels of calcitonin gene-related peptide are lower in COVID-19 patients. J Endocr Soc. 5(3):bvaa199. https:// doi.org/10.1210/jendso/bvaa199

12. Arca KN, Starling AJ (2020) Treatment-refractory headache in the setting of COVID-19 pneumonia: migraine or meningoencephalitis? Case Report. SN Compr Clin Med. https://doi.org/10.1007/ s42399-020-00369-y
13. Tolebeyan AS, Zhang N, Cooper V et al (2020) Headache in patients with severe acute respiratory syndrome coronavirus 2 infection: a narrative review. Headache 60(10):2131-2138. https:// doi.org/10.1111/head.13980

14. Martelletti P, Bentivegna E, Spuntarelli V et al (2021) LongCOVID Headache. SN Compr Clin Med. https://doi.org/10.1007/ s42399-021-00964-7

Publisher's Note Springer Nature remains neutral with regard to jurisdictional claims in published maps and institutional affiliations. 